\title{
О применении интеллектуальных технологий обработки естественного языка и средств виртуальной реальности для поддержки принятия решений при подборе исполнителей проектов
}

\author{
1) Агузумцян Р.В., ${ }^{2)}$ Великанова А.С., ${ }^{2)}$ Польщиков К.А., ${ }^{2)}$ Игитян Е.В., ${ }^{2)}$ Лихошерстов Р.В. \\ 1) Академия государственного управления Республики Армения, \\ Республика Армения, 0028, г. Ереван, ул. Киевян 8 \\ ${ }^{2)}$ Белгородский государственный национальный исследовательский университет, \\ Россия, 308015, г. Белгород, ул. Победы, 85 \\ E-mail: rvaghuzumtsyan@list.ru,agerasimova@bsu.edu.ru,polshchikov@bsu.edu.ru, \\ medvedeva_e@bsu.edu.ru, oaqwater@yandex.ru
}

\begin{abstract}
Аннотация. Представлены концептуальные основы оценивания личностных приоритетов человека и его нацеленности на достижение результатов проекта на основе применения методов нечеткой логики, нейронных сетей и средств виртуальной реальности. Для оценивания нацеленности человека на достижение результатов проекта предложено использовать четырёхслойную нейронно-нечеткую сеть, обученную на экспертных данных об исполнителях ранее реализованных проектов. Выявление личностных приоритетов человека основано на использовании интеллектуального анализа текстовых интернет-сообщений человека с помощью применения нейросетевых технологий обработки естественного языка. Исследования могут быть использованы для создания программных средств поддержки принятия решений при подборе исполнителей о включении человека в состав проектной команды.
\end{abstract}

Ключевые слова: нацеленность на достижение результатов проекта, личностные приоритеты, нейронно-нечеткая сеть, обработка естественного языка, средства виртуальной реальности, принятие решений.

Благодарности: исследование выполнено при финансовой поддержке РФФИ в рамках научного проекта № 20-37-90083.

Для цитирования: Агузумцян Р.В., Великанова А.С., Польщиков К.А., Игитян Е.В., Лихошерстов Р.В. 2021. О применении интеллектуальных технологий обработки естественного языка и средств виртуальной реальности для поддержки принятия решений при подборе исполнителей проектов. Экономика. Информатика, 48 (2): 392-404. DOI 10.52575/2687-0932-2021-48-2-392-404.

\section{Application of intellectual technologies of natural language processing and virtual reality means to support decision-making when selecting project executors}

\author{
${ }^{1)}$ Ruben V. Aguzumtsyan, ${ }^{2)}$ Alexandra S. Velikanova, ${ }^{2)}$ Konstantin A. Polshchikov, ${ }^{2)}$ Elena V. Igityan, \\ ${ }^{2)}$ Rodion V. Likhosherstov \\ 1) Academy of Public Administration of the Republic of Armenia, \\ 8 Kievyan str., Yerevan 0028, Republic of Armenia \\ 2) Belgorod National Research University, 85 Pobeda St, Belgorod, 308015, Russia \\ E-mail: rvaghuzumtsyan@list.ru, agerasimova@bsu.edu.ru,polshchikov@bsu.edu.ru, \\ medvedeva_e@bsu.edu.ru,oaqwater@yandex.ru
}

Abstract. The conceptual foundations of assessing a person's personal priorities and his project results
targeting based on the use of fuzzy logic methods, neural networks and virtual reality tools are presented. To
assess the project results targeting of a person, it is proposed to use a four-layer neural-fuzzy network trained
on expert data on the executors of previously implemented projects. The identification of a person's personal 
priorities is based on the use of intellectual analysis of textual Internet messages of a person using neural network technologies for natural language processing. As a training sample, it is proposed to use a set of text document vectors and the corresponding marks of personal priority classes. In the process of identifying the personal priorities classes, it is required to create an appropriate text array based on parsing and processing of text messages published on the Internet by the analyzed person. Research can be used to create software tools to support decision-making in the selection of performers on the inclusion of a person in the project team.

Keywords: project results targeting, personal priorities, neural-fuzzy network, natural language processing, virtual reality tools, decision-making.

Acknowledgments: the reported study was funded by RFBR, project number 20-37-90083.

For citation: Aguzumtsyan R.V., Velikanova A.S., Polshchikov K.A., Igityan E.V., Likhosherstov R.V. 2021. Application of intellectual technologies of natural language processing and virtual reality means to support decision-making when selecting project executors. Economics. Information technologies, 48 (2): 392-404. (in Russian). DOI 10.52575/2687-0932-2021-48-2-392-404.

\section{Введение}

Органы государственной власти, государственные структуры и коммерческие организации различных отраслей экономики ставят перед собой задачи разработки и реализации значимых проектов. Многочисленные исследования посвящены вопросам совершенствования подходов к управлению государственными проектами и программами. Анализ публикаций в этой сфере показал, что в процессе реализации крупномасштабных проектов могут возникать существенные трудности: нехватка квалифицированных исполнителей, недостаток ресурсов, потребности в выполнении дополнительных работ [Chih, Zwikael, 2015; Zwikael, Smyrk, 2015; Winch, Cha, 2020; Perera, Dewagoda, 2021]. Указанные проблемы в условиях неэффективного управления и отсутствия ответственности со стороны должностных лиц приводят к задержкам, блокировкам проектов и существенному перерасходу средств на их реализацию [Patanakul et al., 2016; Hetemi et al., 2020].

При этом работодатели сталкиваются со сложностями в подборе профессиональных кадров, способных внедрять проекты и доводить начатую деятельность до успешного завершения. Принимать решение о включении лица в проектную команду предлагается с учетом его нацеленности на достижение результатов проекта (Project Results Targeting, PRT) [Gerasimova, 2013; Gerasimova, Oboznov, 2014; Gerasimova, Oboznov, 2015; Ovsyanikova et al., 2018]. Определяющим фактором PRT, а также успешности профессиональной деятельности и психологического благополучия человека являются его личностные приоритеты - цели, ценности и смыслы, присущие внутреннему миру человека, определяющие его мотивационную направленность в жизнедеятельности. Стремление личности к соблюдению моральных норм в поведении приводит его к удовлетворённости своей работой и жизнью в целом [Gerasimova, 2013; Gerasimova, Oboznov, 2015; Ovsyanikova et al., 2018].

Учет PRT и личностных приоритетов позволяет работодателям повысить обоснованность принятия решений в отношении трудоустройства или назначения конкретных лиц на определенные должности в компаниях и организациях [Gerasimova, Oboznov, 2014]. Личностные приоритеты являются скрытыми от внешнего наблюдения, а порой сознательно скрываемыми особенностями человека, которые проявляются в процессе длительного совместного труда и взаимодействия в различных проблемных ситуациях, возникающих в коллективе. Это обстоятельство существенно затрудняет получение информации о личностных приоритетах незнакомых лиц. Для уменьшения нежелательного риска при приеме человека на работу традиционно используются рекомендательные документы от авторитетных лиц, к которым у работодателя имеется высокое доверие. Однако многие не имеют возможности получить такие рекомендации, например, из-за незначительного опыта работы или его отсутствия. Необходимость разработки новых средств оценки PRT и личностных приоритетов человека определяет актуальность исследований, представленных в данной статье. 
Цель данного исследования состоит в формировании концептуальных основ применения технологий искусственного интеллекта и виртуальной реальности для поддержки принятия решений о включении человека в состав проектной команды на основе оценивания его личностных приоритетов и нацеленности на достижение результатов проекта.

\section{Применение средств виртуальной реальности и нечеткой нейронной сети для выявления нацеленности человека на достижение результатов проекта}

С целью выявления нацеленности человека на достижение результатов проекта предлагается создать и использовать VR-сценарии, т. е. визуализированные с помощью средств виртуальной реальности проблемные ситуации. Рекомендуется применять два набора VR-сценариев (VR-ситуаций) для оценивания PRT испытуемого. Сценарии из набора 1 должны быть предназначены для оценивания величины $S-$ устойчивости PRT-позиции испытуемого. Сценарии из набора 2 должны быть предназначены для оценивания величины $A$ - склонности испытуемого к РRT-действиям. Для вычисления величины $S$ можно использовать выражение:

$$
S=\sum_{i=1}^{I} k_{i} s_{i},
$$

где $I$ - число VR-сценариев в наборе $1 ; i$ - номер VR-сценария в наборе $1, i=1,2, \ldots, I ; k_{i}-$ весовой коэффициент VR-сценария номер $i$ в наборе $1 ; k_{i}$ может принимать значения от 0 до $1 ; s_{i}$ - индикатор принятия испытуемым PRT-позиции в VR-ситуации номер $i$ в наборе 1; $s_{i}=1$ в случае принятия испытуемым PRT-позиции, $s_{i}=0$ в противном случае.

Вычисление величины $A$ может быть выполнено с использованием выражения:

$$
A=\sum_{j=1}^{J} h_{j} a_{j},
$$

где $J$ - число VR-сценариев в наборе $2 ; j$ - номер VR-сценария в наборе $2, j=1,2, \ldots, J ; h_{j}-$ весовой коэффициент VR-сценария номер $j$ в наборе $2 ; h_{j}$ может принимать значения от 0 до $1 ; a_{j}$ - индикатор выполнения испытуемым PRT-действий в VR-ситуации номер $j$ в наборе 2 ; $a_{j}=1$ в случае выполнения испытуемым PRT-действий, $a_{j}=0$ в противном случае.

Для установления значений весовых коэффициентов $k_{i}$ и $h_{j}$ предлагается применить экспертные оценки значимости конкретных VR-сценариев для выявления устойчивости PRTпозиции испытуемого и его склонности к PRT-действиям. С этой целью от каждого из $N$ экспертов необходимо получить индивидуальные оценки $e_{n i}$ и $r_{n j}$ каждого используемого VR-сценария. Полученные значения экспертных оценок должны быть занесены в две базы данных, сформированные в соответствии с таблицами 1 и 2.

Таблица 1

Table 1

Значения экспертных оценок для установления значений весовых коэффициентов $k_{i}$

Values of expert assessments for establishing the values of weighting coefficients $k_{i}$

\begin{tabular}{|c|c|c|c|c|}
\hline \multirow{2}{*}{ Номер эксперта } & \multicolumn{4}{|c|}{ Номер VR-сценария в наборе 1 } \\
\cline { 2 - 5 } & 1 & 2 & $\ldots$ & $I$ \\
\hline 1 & $e_{11}$ & $e_{12}$ & $\ldots$ & $e_{1 I}$ \\
\hline 2 & $e_{21}$ & $e_{22}$ & $\ldots$ & $e_{2 I}$ \\
\hline$\ldots$ & $\ldots$ & $\ldots$ & $\ldots$ & $\ldots$ \\
\hline$N$ & $e_{N 1}$ & $e_{N 2}$ & $\ldots$ & $e_{N I}$ \\
\hline
\end{tabular}


Значения экспертных оценок для установления значений весовых коэффициентов $h_{j}$

Values of expert assessments for establishing the values of weighting coefficients $h_{j}$

\begin{tabular}{|c|c|c|c|c|}
\hline \multirow{2}{*}{ Номер эксперта } & \multicolumn{4}{|c|}{ Номер VR-сценария в наборе 2 } \\
\cline { 2 - 5 } & 1 & 2 & $\ldots$ & $J$ \\
\hline 1 & $r_{11}$ & $r_{12}$ & $\ldots$ & $r_{1 J}$ \\
\hline 2 & $r_{21}$ & $r_{22}$ & $\ldots$ & $r_{2 J}$ \\
\hline$\ldots$ & $\ldots$ & $\ldots$ & $\ldots$ & $\ldots$ \\
\hline$N$ & $r_{N 1}$ & $r_{N 2}$ & $\ldots$ & $r_{N J}$ \\
\hline
\end{tabular}

При выставлении оценок $e_{n i}$ и $r_{n j}$ эксперты могут использовать значения десятибалльной шкалы. На основе данных, содержащихся в таблицах 1 и 2, можно вычислить средние экспертные оценки VR-сценария номер $i$ в наборе 1 и VR-сценария номер $j$ в наборе 2:

$$
\begin{gathered}
E_{i}=\frac{\sum_{n=1}^{N} e_{n i}}{N}, \\
R_{j}=\frac{\sum_{n=1}^{N} r_{n j}}{N} .
\end{gathered}
$$

Тогда для вычисления значений весовых коэффициентов $k_{i}$ и $h_{j}$ можно применить следующие формулы:

$$
\begin{aligned}
& k_{i}=\frac{E_{i}}{\sum_{i=1}^{I} E_{i}}, \\
& h_{j}=\frac{R_{j}}{\sum_{j=1}^{J} R_{j}} .
\end{aligned}
$$

Для получения результирующей оценки степени альтруизма кандидата (претендента) введем обобщенный показатель $D$, учитывающий частные показатели устойчивости PRT-позиции кандидата и его склонности к PRT-действиям, т. е. величины $S$ и $A$.

Пожалуй, невозможно точно определить конкретный интервал численных значений величины $S$, при которых РRT-позиция человека однозначно является устойчивой. Аналогично, весьма проблематично точно определить численные границы значений величины $A$, в пределах которых человека можно однозначно отнести к приверженцам PRT-действий. В связи с этим для оценивания величины $S$ можно использовать нечеткие множества (термы) «высокая устойчивость РRT-позиции» и «низкая устойчивость PRT-позиции», а для оценивания величины $A$ можно использовать термы «высокая склонность к PRT-действиям» и «низкая склонность к PRT-действиям». В таком случае, чтобы вычислить показатель $D$, можно воспользоваться нечеткими правилами простейшего вида, соответствующими алгоритму нечеткого вывода Сугено нулевого порядка:

$$
\begin{aligned}
& \text { If }\left(S=S^{+}\right) \text {and }\left(A=A^{+}\right) \text {then }\left(D=d_{1}\right) \text {, } \\
& \text { If }\left(S=S^{+}\right) \text {and }\left(A=A^{-}\right) \text {then }\left(D=d_{2}\right) \text {, }
\end{aligned}
$$




$$
\begin{aligned}
& \text { If }\left(S=S^{-}\right) \text {and }\left(A=A^{+}\right) \text {then }\left(D=d_{3}\right), \\
& \text { If }\left(S=S^{-}\right) \text {and }\left(A=A^{-}\right) \text {then }\left(D=d_{4}\right),
\end{aligned}
$$

где $S^{+}$- терм «высокая устойчивость PRT-позиции»; $S^{-}-$терм «низкая устойчивость PRT-позиции»; $A^{+}$- терм «высокая склонность к PRT-действиям»; $A^{-}$- терм «низкая склонность к PRT-действиям»; $d_{1}, d_{2}, d_{3}$ и $d_{4}$ - значения индивидуальных выводов соответствующих нечетких правил.

Значения $S$ могут в большей или меньшей мере соответствовать термам $S^{+}$и $S^{-}$. Для вычисления величины этого соответствия воспользуемся функциями принадлежности $x^{+}(S)$ и $x^{-}(S)$. Их смысл заключается в том, что значение функции $x^{+}(S)$ показывает, с какой вероятностью значение $S$ принадлежит терму «высокая устойчивость PRT-позиции», а значение функции $x^{-}(S)$ показывает, с какой вероятностью значение $S$ принадлежит терму «низкая устойчивость PRT-позиции». Аналогично для вычисления соответствия значений $A$ термам $A^{+}$и $A^{-}$можно использовать функции принадлежности $y^{+}(A)$ и $y^{-}(A)$. Широкое применение в исследовательской практике получили кусочно-непрерывные функции принадлежности [Polshchykov et al., 2019; Polshchykov et al., 2020], имеющие вид, представленный на рисунках 1 и 2.
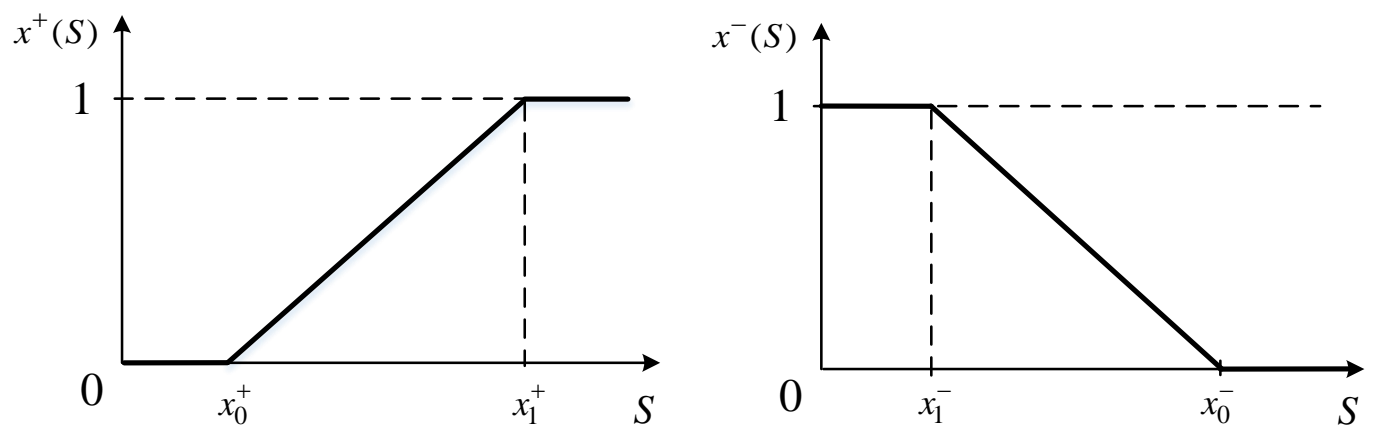

Рис. 1. Функции принадлежности $x^{+}(S)$ и $x^{-}(S)$

Fig. 1. Membership functions $x^{+}(S)$ and $x^{-}(S)$
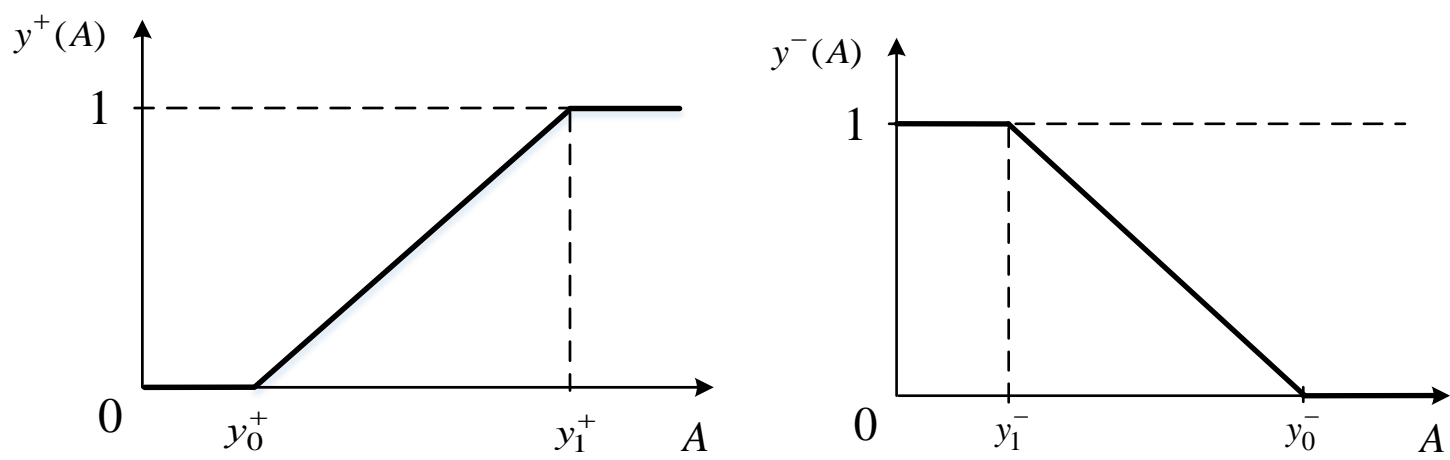

Рис. 2. Функции принадлежности $y^{+}(A)$ и $y^{-}(A)$

Fig. 2. Membership functions $y^{+}(A)$ and $y^{-}(A)$ 
На рисунках 1 и 2 обозначены следующие величины: $x_{0}^{+}$и $x_{1}^{+}-$границы наклонного отрезка ломаной $x^{+}(S) ; x_{1}^{-}$и $x_{0}^{-}$- границы наклонного отрезка ломаной $x^{-}(S) ; y_{0}^{+}$и $y_{1}^{+}-$ границы наклонного отрезка ломаной $y^{+}(A) ; y_{1}^{-}$и $y_{0}^{-}$-границы наклонного отрезка ломаной $y^{-}(A)$.

Чтобы найти показатель $D$ на основе нечетких правил (7)-(10), прежде всего, необходимо выполнить фаззификацию, т. е. вычислить значения функций принадлежности величины $S$ термам $S^{+}$и $S^{-}$, а также значения функций принадлежности величины $A$ термам $A^{+}$и $A^{-}$:

$$
\begin{aligned}
& x^{+}(S)=\left\{\begin{array}{l}
0, S<x_{0}^{+} ; \\
\frac{S-x_{0}^{+}}{x_{0}^{+}-x_{1}^{+}}, x_{0}^{+} \leq S<x_{1}^{+} ; \\
1, S \geq x_{1}^{+} ;
\end{array}\right. \\
& x^{-}(S)=\left\{\begin{array}{l}
1, S<x_{1}^{-} ; \\
\frac{x_{0}^{-}-S}{x_{0}^{-}-x_{1}^{-}}, x_{1}^{-} \leq S<x_{0}^{-} ; \\
0, S \geq x_{0}^{-} ;
\end{array}\right. \\
& y^{+}(A)=\left\{\begin{array}{l}
0, A<y_{0}^{+} ; \\
\frac{A-y_{0}^{+}}{y_{0}^{+}-y_{1}^{+}}, x_{0}^{+} \leq A<x_{1}^{+} ; \\
1, A \geq y_{1}^{+} ;
\end{array},\right. \\
& y^{-}(A)=\left\{\begin{array}{l}
1, A<y_{0}^{-} ; \\
\frac{y_{0}^{-}-A}{y_{0}^{-}-y_{1}^{-}}, y_{1}^{-} \leq A<y_{0}^{-} ; \\
0, A \geq y_{0}^{-} ;
\end{array}\right.
\end{aligned}
$$

Следующим этапом является агрегирование:

$$
\begin{aligned}
& G_{1}=x^{+}(S) \wedge y^{+}(A), \\
& G_{2}=x^{+}(S) \wedge y^{-}(A), \\
& G_{3}=x^{-}(S) \wedge y^{+}(A), \\
& G_{4}=x^{-}(S) \wedge y^{-}(A) .
\end{aligned}
$$

Заключительным этапом оценивания PRT является дефаззификация, в результате которой вычисляется показатель $D$ по формуле:

$$
D=\frac{G_{1} d_{1}+G_{2} d_{2}+G_{3} d_{3}+G_{4} d_{4}}{G_{1}+G_{2}+G_{3}+G_{4}} .
$$

В представленных выше выражениях (11)-(14) и (19) неизвестными остаются значения параметров $d_{1}, d_{2}, d_{3}, d_{4}, x_{0}^{+}, x_{1}^{+}, x_{1}^{-}, x_{0}^{-}, y_{0}^{+}, y_{1}^{+}, y_{1}^{-}$и $y_{0}^{-}$, для вычисления которых можно воспользоваться возможностями обучения многослойной нейронной сети. В данном случае 
следует создать четырехслойную нейронную сеть (рис. 3). В ней каждый слой предназначен для выполнения определенных процедур нечеткого вывода. Такую гибридную структуру именуют нейронно-нечеткой сетью или ANFIS (Adaptive-Network-Based Fuzzy Inference System) [Polshchykov et al., 2015; Konstantinov et al., 2017; Polshchykov et al., 2017; Feng, Chen, 2018; Škrjanc et al., 2019; Shihabudheen, Pillai, 2019].

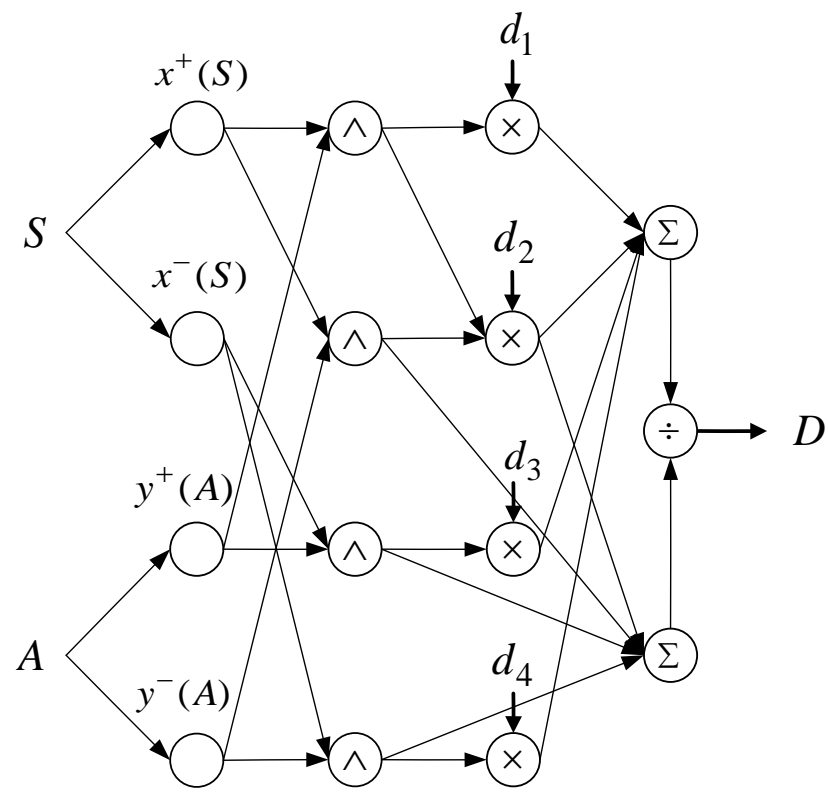

Рис. 3. Структура нейронно-нечеткой сети, предназначенной для оценки PRT

Fig 3. Structure of a neural-fuzzy network designed for PRT estimation

Чтобы настроить такую нейронно-нечеткую сеть, потребуется обучающая выборка, которая может быть представлена в виде данных таблицы 3.

Состав данных обучающей выборки

The composition of the training sample data

\begin{tabular}{|c|c|c|c|}
\hline $\begin{array}{c}\text { Порядковый номер } m \\
\text { испытуемого }\end{array}$ & $\begin{array}{c}\text { Значение } \\
\text { оценки } S_{m}\end{array}$ & Значение оценки $A_{m}$ & $\begin{array}{c}\text { Значение показателя } \\
D_{m}\end{array}$ \\
\hline 1 & $S_{1}$ & $A_{1}$ & $D_{1}$ \\
\hline 2 & $S_{2}$ & $A_{2}$ & $D_{2}$ \\
\hline$\ldots$ & & & $D_{M}$ \\
\hline$M$ & $S_{M}$ & $A_{M}$ & \\
\hline
\end{tabular}

Число строк в структуре обучающей выборки соответствует числу испытуемых лиц $M$. В качестве испытуемых лиц для формирования обучающей выборки предлагается привлечь участников команд реализованных проектов, степень PRT которых могут оценить другие члены этих же проектных команд, например, коллеги вышестоящего уровня. Оценка PRT $D_{m}$ испытуемого $m$ может быть вычислена с помощью выражения:

$$
D_{m}=\frac{\sum_{v=1}^{V} q_{m v}}{V},
$$

где $V$ - число лиц, давших оценку PRT испытуемого $m ; q_{m v}$ - оценка PRT испытуемого $m$, данная лицом $v$. 
При выставлении оценок $q_{m v}$ могут использоваться значения десятибалльной шкалы.

В таблице 3 каждое значение $S_{m}$ - это оценка устойчивости PRT-позиции испытуемого $m$, а каждое значение $A_{m}$ - это оценка склонности испытуемого $m$ к PRT-действиям. Для получения этих оценок каждому лицу из числа $M$ потребуется пройти серию испытаний с использованием средств виртуальной реальности. В результате проведения испытаний будут получены реакции $s_{m i}$ и $a_{m j}$ каждого испытуемого лица $m$ в каждой конкретной VR-ситуации из наборов 1 и 2. Затем значения $S_{m}$ и $A_{m}$ могут быть вычислены по формулам:

$$
\begin{gathered}
S_{m}=\sum_{i=1}^{I} k_{i} s_{m i}, \\
A_{m}=\sum_{j=1}^{J} h_{j} a_{m j},
\end{gathered}
$$

где $s_{m i}-$ индикатор принятия испытуемым $m$ PRT-позиции в VR-ситуации номер $i$ в наборе 1 ( $s_{m i}=1$ в случае принятия испытуемым PRT-позиции, $s_{m i}=0$ в противном случае); $a_{m j}$ - индикатор выполнения испытуемым $m$ PRT-действий в VR-ситуации номер $j$ в наборе 2 ( $a_{m j}=1$ в случае выполнения испытуемым PRT-действий, $a_{m j}=0$ в противном случае).

Полученная таким образом обучающая выборка может быть использована для настройки нейронно-нечеткой сети. Обучение ANFIS, как правило, выполняется с помощью быстрого алгоритма обратного распространения ошибки или гибридного алгоритма, усложненного применением метода наименьших квадратов. В ходе многократных циклов обучения (эпох) осуществляется настройка весов нейронов первого и третьего слоёв ANFIS. Настройку необходимо выполнять до тех пор, пока значение ошибки обучения не стабилизируется на минимальном уровне. В результате настройки весов нейронов первого слоя получим искомые значения $x_{0}^{+}, x_{1}^{+}, x_{1}^{-}, x_{0}^{-}, y_{0}^{+}, y_{1}^{+}, y_{1}^{-}$и $y_{0}^{-}$. Искомые значения $d_{1}, d_{2}, d_{3}$ и $d_{4}$ будут соответствовать весам нейронов третьего слоя обученной нейронно-нечеткой сети.

После настройки ANFIS-систему можно использовать для поддержки принятия решения о включении в проектную команду того или иного кандидата с учетом его PRT. Вычисленные по формулам (1) и (2) показатели устойчивости PRT-позиции кандидата и его склонности к PRT-действиям следует использовать в качестве входных величин нейроннонечеткой сети. В слоях ANFIS будут выполнены вычисления в соответствии с выражениями (11)-(19), результатом которых на выходе сети станет оценка $D$ - обобщенный показатель PRT кандидата на включение в проектную команду. Исходя из предлагаемой концепции, лица с высокими значениями $D$ будут в первую очередь рекомендованы ко включению в коллектив исполнителей проекта.

\section{Выявление личностных приоритетов на основе интеллектуальных средств обработки естественного языка}

В качестве дополнительного средства подбора исполнителей значимых проектов предлагается использовать выявление личностных приоритетов на основе интеллектуального анализа цифровых следов и текстового контента на общедоступных ресурсах интернетмессенджеров, чатов и социальных сетей. В современном обществе характерная особенность коммуникации состоит в массовом активном использовании Интернет-сервисов. Пользователи цифровых гаджетов ежедневно генерируют в Интернет-ресурсах огромное количество текстовых и голосовых сообщений, твиттов, микроблогов, комментариев. Эти данные могут быть использованы для получения необходимой информации об интересах конкретного 
человека, о его личных качествах и позиции по отношению к различным явлениям в социальной, культурной, политической и экономической сферах общественной жизни.

На основе классификации жизненных ценностей можно выделить типы личностных приоритетов, определяющих профессиональное и экономическое благополучие человека. Первый тип именуется нравственно-деловым (moral and business priorities, MBP). Для него характерны преобладание духовно-нравственных, гуманистических ценностей, приверженность к соблюдению нравственных норм в поведении, коллективизму, готовности помогать и поддерживать других людей, к развитию себя с целью обеспечения общественного благополучия. Второй тип личностных приоритетов - эгоистически-престижный (selfishprestigious priorities, SPP). Человек с таким типом личностных приоритетов отдает предпочтение прагматическим ценностям, индивидуализму, собственному престижу, личным достижениям, высокому материальному положению, эгоистическому выделению собственной персоны.

Выявление личностных приоритетов анализируемого лица сводится к решению задачи классификации, т. е. к отнесению личностных приоритетов человека к классу SPP или MBP. Эту задачу предлагается решить на основе анализа текстовых интернет-следов человека с помощью применения нейросетевых технологий обработки естественного языка (Natural Language Processing, NLP) [Young et al., 2018]. Интеллектуальные средства обработки естественно-языковых данных успешно применяются для решения многих практических задач [Öztürk et al., 2020; Stewart, Velupillai, 2021; Arts et al., 2021], поэтому вполне обоснованной является попытка использовать NLP с целью выявления личностных приоритетов.

Для реализации этой идеи, прежде всего, необходимо отобрать $K$ человек, классы личностных приоритетов которых заранее определены на основе экспертных оценок других людей, например, их руководителей, сослуживцев, партнеров и т. п. На следующем этапе требуется выполнить парсинг интернет-сообщений, опубликованных каждым отобранным лицом, и сформировать из них $K$ сборных текстовых массивов.

Затем каждый полученный текстовый массив следует подвергнуть подготовительной NLP-обработке, выполнив процедуры сегментации текста на предложения, их токенизации на отдельные слова, исключения стоп-слов и знаков препинания, лемматизации. На следующем шаге для каждого документа номер $k$ на основе использования статистического метода для оценки важности слов в документе (Term Frequency - Inverse Document Frequency, TF-IDF) [Kim et al., 2019] необходимо составить вектор $C_{k}$, формат которого представлен на рисунке 4.

\begin{tabular}{|l|l|l|l|l|l|l|}
\hline$c_{1}$ & $c_{2}$ & $c_{3}$ & $\cdots$ & $c_{w}$ & $\cdots$ & $c_{Z}$ \\
\hline
\end{tabular}

Рис. 4. Формат вектора текстового документа

Fig 4. Text document vector format

Значения элементов $c_{w}$ вектора текстового документа вычисляются с помощью выражения:

$$
c_{w}=\frac{u_{w}}{\sum_{z=1}^{Z} u_{z}} \log \frac{K}{F_{w}},
$$

где $u_{w}$ - количество слов номер $w$ в текстовом документе; $Z$ - число слов в используемом словаре; $u_{z}-$ количество слов номер $z$ в текстовом документе; $F_{w}$ - число текстовых документов, в которых содержится слово номер $w$.

Чтобы на основе вектора текстового документа определить, к какому классу (SPP или MBP) относятся личностные приоритеты лица, опубликовавшего соответствующий набор интернет-сообщений, предлагается использовать возможности нейронной сети. Для решения 
задач NLP чаще всего применяются рекуррентные нейронные сети (Recurrent Neural Networks, RNN) [Pitsilis et al., 2018], сети с долгой краткосрочной памятью (Long Short-Term Memory, LSTM) [Qaisar, 2020], а также сети-трансформеры (Generative Pre-trained Transformer, GPT-2, GPT-3) [Lee et al., 2020; Dehouche, 2021]. При этом требуется обучение нейронной сети, в результате которого параметры нейронов настраиваются таким образом, чтобы ошибки классификации были минимизированы.

В качестве обучающей выборки предлагается использовать набор из $K$ векторов текстовых документов и соответствующих им меток классов личностных приоритетов. Метка $l_{k}$ принимает значение 0, если личностные приоритеты человека относятся к классу SPP. Метка $l_{k}$ равна 1 , если личностные приоритеты человека относятся к классу МВР. Пример фрагментов обучающей выборки выделен серым цветом в таблице 4.

Таблица 4

Table 4

Формат и пример фрагментов обучающей выборки

Format and example of training sample fragments

\begin{tabular}{|c|c|c|c|c|c|c|c|}
\hline \multirow{3}{*}{$\begin{array}{c}\text { Номер } \\
\text { текстового } \\
\text { документа }\end{array}$} & \multicolumn{7}{|c|}{ Элементы обучающей выборки } \\
\hline & \multicolumn{6}{|c|}{ Элементы текстового документа } & \multirow{2}{*}{$\begin{array}{c}\text { Метки классов } \\
\text { личностных } \\
\text { приоритетов } \\
l_{k}\end{array}$} \\
\hline & $c_{1}$ & $c_{2}$ & ... & $c_{w}$ & ... & $c_{Z}$ & \\
\hline 1 & 0 & 0,0024 & $\ldots$ & 0 & $\ldots$ & 0,0018 & 1 \\
\hline 2 & 0 & 0 & $\ldots$ & 0,0004 & $\ldots$ & 0 & 0 \\
\hline$\ldots$ & $\ldots$ & $\ldots$ & $\ldots$ & $\ldots$ & $\ldots$ & $\ldots$ & $\ldots$ \\
\hline$k$ & 0 & 0 & $\ldots$ & 0,0002 & $\ldots$ & 0,0012 & 1 \\
\hline$\ldots$ & $\ldots$ & $\ldots$ & $\ldots$ & $\ldots$ & $\ldots$ & $\ldots$ & $\ldots$ \\
\hline$K$ & 0,0001 & 0 & $\ldots$ & 0 & $\ldots$ & 0 & 0 \\
\hline
\end{tabular}

После настройки нейронная сеть может быть использована для определения класса личностных приоритетов анализируемого лица. С этой целью с помощью парсинга открытых сетевых ресурсов потребуется собрать опубликованные этим человеком интернет-сообщения в текстовый массив, провести его подготовительную NLP-обработку и сформировать вектор соответствующего текстового документа с элементами, значения которых вычислены по формуле (23). Затем сформированный вектор потребуется подать на вход ранее обученной нейронной сети (рис. 5).

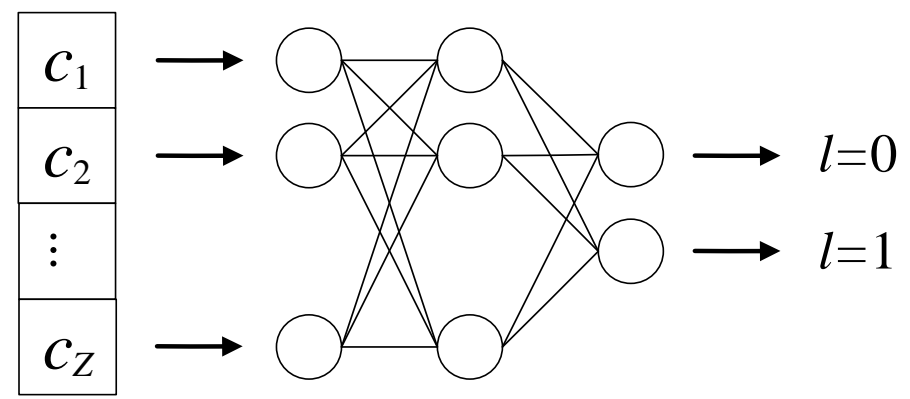

Рис. 5. Входные и выходные величины нейронной сети

Fig 5. Input and output values of the neural network 
В результате на выходе нейронной сети будет получена метка класса личностных приоритетов анализируемого лица. В соответствии с предлагаемой концепцией в коллектив исполнителей проекта в первую очередь будут рекомендованы лица, отнесенные к классу МВР.

\section{Заключение}

Таким образом, предложены концептуальные основы применения технологий искусственного интеллекта и виртуальной реальности для поддержки принятия решений при подборе кандидатов в состав проектной команды на основе оценивания личностных приоритетов и нацеленности на достижение результатов проекта.

Показатели устойчивости PRT-позиции кандидата и его склонности к PRT-действиям следует использовать в качестве входных величин нейронно-нечеткой сети. Слоями нейронов ANFIS выполняются функции фаззификации, агрегирования и дефаззификации, в результате которых на выходе сети вычисляется оценка D - обобщенный показатель PRT кандидата на включение в проектную команду. Исходя из предлагаемой концепции, лица с высокими значениями D будут в первую очередь рекомендованы ко включению в коллектив исполнителей проекта.

В качестве дополнительного средства подбора исполнителей значимых проектов предлагается использовать интеллектуальный анализ текстовых сообщений, публикуемых интернет-пользователями, для определения личностных приоритетов кандидатов. С точки зрения эффективности выполнения человеком задач в трудовом коллективе всё многообразие личностных приоритетов может быть сведено к двум основным классам SPP и МВР. Процесс выявления классов личностных приоритетов предполагает выполнение следующих этапов:

1) на основе парсинга и NLP-обработки текстовых сообщений, опубликованных в Интернете анализируемым лицом, составляется соответствующий текстовый массив;

2) из полученного текстового массива формируется текстовый вектор документа, элементы которого вычисляются с помощью метода TF-IDF;

3) текстовый вектор документа подается на вход нейронной сети, благодаря настройке которой в выходном слое нейронов отображается метка класса личностных приоритетов анализируемого лица.

Предметом дальнейших исследований станет обоснование выбора типа и параметров нейронной сети для решения задачи классификации личностных приоритетов. Кроме того, потребуется разработка программных средств для реализации предложенных в работе концептуальных основ и получения экспериментальных результатов, позволяющих оценить возможности выявления у человека личностных приоритетов и нацеленности на достижение результатов проекта.

\section{References}

1. Arts S., Hou J., Gomez J.C. 2021. Natural language processing to identify the creation and impact of new technologies in patent text: Code, data, and new measures. Research Policy, 50: 104-144.

2. Chih Y., Zwikael O. 2015. Project benefit management: A conceptual framework of target benefit formulation. International Journal of Project Management, 33: 352-362.

3. Dehouche N. 2021. Plagiarism in the age of massive Generative Pre-trained Transformers (GPT3). Ethics Sci Environ Polit, 21: 17-23.

4. Feng S., Chen C.L.P. 2018. Fuzzy broad learning system: A novel neuro-fuzzy model for regression and classification. IEEE Transactions on Cybernetics, 50: 414-424.

5. Gerasimova A., Oboznov A. 2014. Development of an altruistic orientation of the personality of students and expert of helping professions. International Multidisciplinary Scientific Conferences on Social Sciences and Arts. Psychology and Psychiatry, Sociology and Healthcare, Education, 1: 115-120.

6. Gerasimova A., Oboznov A. 2015. Normative orientation of the specialists of official activity and professionals of socionomic profile Proceedings of 2nd Global Conference on Psychology Researches (GCPR2014). Procedia Social and Behavioral Sciences, 190: 39-42. 
7. Gerasimova A.S. 2013. Value-normative method of evaluation of educational motivation of students. Eksperimentalnaya psikhologiya, 6: 96-104.

8. Hetemi E., Jerbrant A., Mereb J. O. 2020. Exploring the emergence of lock-in in large-scale projects: A process view. International Journal of Project Management, 38: 47-63.

9. Kim D., Seo D., Cho S., Kang P. 2019. Multi-co-training for document classification using various document representations: TF-IDF, LDA, and Doc2Vec. Inform Sciences, 477: 15-29.

10.Konstantinov I., Polshchykov K., Lazarev S., Polshchykova O. 2017. Model of Neuro-Fuzzy Prediction of Confirmation Timeout in a Mobile Ad Hoc Network. CEUR Workshop Proceedings. Mathematical and Information Technologies, 1839: 174-186.

11.Konstantinov I.S., Polshchykov K.O., Lazarev S.A. 2017. The Algorithm for Neuro-Fuzzy Controlling the Intensity of Retransmission in a Mobile Ad-Hoc Network. International Journal of Applied Mathematics and Statistics, 56: 85-90.

12.Lee H.H., Shu K., Achananuparp P., Prasetyo P K., Liu Y., Lim E.-P., Varshney L.R. 2020. Generative Pre-training Based Cooking Recipe Generation and Evaluation System. WWW'20: Companion Proceedings of the Web Conference: 181-184.

13.Ovsyanikova E.A., Mandibura N.A., Gerasimova A.S., Hudayeva M.Y., Tkachenko N.S., Godovnikova L.V. 2018. Modern status of research on the problem of psychological well-being of the person in the domestic and world psychological science. Revista Publicando, 5: 349-358.

14.Öztürk H., Özgür A., Schwaller P., Laino T., Ozkirimli E. 2020. Exploring chemical space using natural language processing methodologies for drug discovery. Drug Disc Today, 25: 689-705.

15.Patanakul P., Kwak Y.H., Zwikael O., Liu M. 2016. What Impacts the Performance of Large-Scale Government Projects? International Journal of Project Management, 34: 452-466.

16.Perera B.A.K.S., Dewagoda K.G. 2021. Streamlining the management of payment delays: the case of Sri Lankan Government building construction projects Journal of Financial Management of Property and Construction. Available at: https://doi.org/10.1108/JFMPC-05-2020-0041 (accessed 20 May2021).

17.Pitsilis G. K., Ramampiaro H., Langseth H. 2018. Effective hate-speech detection in Twitter data using recurrent neural networks. Applied Intelligence, 48: 4730-4742.

18.Polshchykov K.A., Lazarev S.A., Konstantinov I.S., Polshchykova O.N., Svoikina L.F., Igityan E.V., Balakshin M.S. 2020. Assessing the Efficiency of Robot Communication. Russian Engineering Research, 40: 936-938.

19.Polshchykov K., Lazarev S., Polshchykova O., Igityan E. 2019. The Algorithm for DecisionMaking Supporting on the Selection of Processing Means for Big Arrays of Natural Language Data. Lobachevskii Journal of Mathematics, 40: 1831-1836.

20.Polshchykov K.O., Lazarev S.A., Zdorovtsov A.D. 2017. Neuro-Fuzzy Control of Data Sending in a Mobile Ad Hoc Network. Journal of Fundamental and Applied Sciences, 9: 1494-1501.

21.Polshchykov K., Zdorenko Y., Masesov M. 2015. Neuro-Fuzzy System for Prediction of Telecommunication Channel Load. Second International Scientific-Practical Conference "Problems of Infocommunications Science and Technology (PIC S\&T)": 33-34.

22.Qaisar S.M. 2020. Sentiment Analysis of IMDb Movie Reviews Using Long Short-Term Memory. 2nd International Conference on Computer and Information Sciences (ICCIS): 1-4.

23. Shihabudheen K.V., Pillai G.N. 2018. Recent advances in neuro-fuzzy system: A survey. Author links open overlay panel. Knowledge-Based Systems, 152: 136-162.

24.Škrjanc I., Iglesias J.A., Sanchis A., Leite D., Lughofer E., Gomide F. 2019. Evolving fuzzy and neuro-fuzzy approaches in clustering, regression, identification, and classification: A Survey. Information Sciences, 490: 344-368.

25.Stewart R., Velupillai S. 2021. Applied natural language processing in mental health big data. Neuropsychopharmacology, 46: 252-253.

26.Winch G.M., Cha J. 2020. Owner challenges on major projects: The case of UK government. International Journal of Project Management, 38: 177-187.

27. Young T., Hazarika D., Poria S., Cambria E. 2018. Recent Trends in Deep Learning Based Natural Language. Processing IEEE Computational Intelligence Magazine, 13: 55-75.

28.Zwikael O., Smyrk J. 2015. Project governance: Balancing control and trust in dealing with risk. International Journal of Project Management, 33: 852-862. 


\section{ИНФОРМАЦИЯ ОБ АВТОРАХ}

Агузумцян Рубен Вазгенович, кандидат психологических наук, профессор, академик Инженерной академии Республики Армения, профессор кафедры психологии управления Академии государственного управления Республики Армения, г. Ереван, Республика Армения

Великанова (Герасимова) Александра Сергеевна, кандидат психологических наук, доцент, доцент кафедры возрастной и социальной психологии Белгородского государственного национального исследовательского университета, г. Белгород, Россия

Польщиков Константин Александрович, доктор технических наук, доцент, директор института инженерных и цифровых технологий Белгородского государственного национального исследовательского университета, г. Белгород, Россия

Игитян Елена Владимировна, аспирант кафедры информационно-телекоммуникацион-ных систем и технологий Белгородского государственного национального исследовательского университета, г. Белгород, Россия

Лихошерстов Родион Валерьевич, соискатель кафедры прикладной информатики и информационных технологий Белгородского государственного национального исследовательского университета, г. Белгород, Россия

\section{INFORMATION ABOUT THE AUTHORS}

Ruben V. Aguzumtsyan, Candidate of Psychological Sciences, Professor, Academician of the Engineering Academy of the Republic of Armenia, Professor of the Department of Management Psychology of the Academy of Public Administration of the Republic of Armenia, Yerevan, Republic of Armenia

Alexandra S. Velikanova (Gerasimova), Candidate of Psychological Sciences, Associate Professor, Associate Professor of the Department of Age and Social Psychology of the Belgorod State National Research University, Belgorod, Russia

Konstantin A. Polshchikov, Doctor of Technical Sciences, Associate Professor, Director of the Institute of Engineering and Digital Technologies of the Belgorod State National Research University, Belgorod, Russia

Elena V. Igityan, Post-graduate Student of the Department of Information and Telecommunications Systems and Technologies of the Belgorod State National Research University, Belgorod, Russia

Rodion V. Likhosherstov, Candidate of the Department of Applied Informatics and Information Technologies of the Belgorod State National Research University, Belgorod, Russia 